\title{
The Integration and Development of Chinese Style Illustrations and Western Painting Aesthetics
}

\author{
Lingxin $\mathrm{Li}^{1{ }^{1, *}}$ Yanjun Wang ${ }^{1}$
}

\author{
${ }^{1}$ Xiamen Academy of Arts and Design, Fuzhou University, Xiamen, Fujian, China \\ *Corresponding author. Email: 200885939@qq.com
}

\begin{abstract}
By virtue of its unique understanding and expression of beauty, Chinese style aesthetics has deeply influenced the aesthetic trend in Asia, as well as survived and thrived against the popular Western aesthetics. The increasingly frequent cross-cultural communication makes the integration of Chinese style illustration and Western painting techniques a new trend. Based on the analysis of representative works of famous Chinese style illustrators at present, this paper discusses how they comprehensively present Chinese elements and Western painting methods, so as to provide reference and insight for designers in exploring the direction of illustration creation.
\end{abstract}

\section{Keywords: Chinese style, illustration design, western aesthetics}

\section{INTRODUCTION}

Chinese painting style enjoys long history, and characteristics varying from dynasties. History has witnessed the division and reorganization of nations, the progress of new technologies and media, and the change of ideologies. All these prompt the continuous development and progress of Chinese painting style, which, like an inexhaustible treasure, serves as the source of inspiration for the art design industry. Artistic language refers to the symbol system employed in various kinds of artistic creation, as well as materialized media through which artists can kindle artistic conception and create works [1]. Illustration design, like traditional painting, expresses itself through artistic language such as color, line, shape and composition. The development of the illustration design industry prompts more and more designers to devote themselves to Chinese illustration, thus vitalizing Chinese aesthetics. Chinese aesthetics are endowed with modern significance through the exchange of ideas and expression with contemporary and past artists in an open cultural environment. Representatives include Victo Ngai, Ruan Feifei, Chinadaily's team, etc. As creators who value tradition but not rigidly adhere to tradition, they carry forward Chinese aesthetics through inheritance.

\section{DIVERSIFIED COMPOSITION}

The composition of traditional Chinese paintings is distinctive. Traditional Chinese paintings adopt the method of scattered perspective. Both vertical landscape and horizontal scroll reflect creators' breakthrough in space and time in traditional Chinese painting. Such free expression is manifested in landscape and delicate position, which mainly includes panoramic space, segmented space and stratified space. Panoramic space refers to rotary and reciprocating flowing space from high to low and from far to near. [2] The composition methods include "zigzag", "tri-line", "diagonal", "triangle", "rectangle", "segmental" and "layering", etc. All these are often seen in modern Chinese illustrations. "Segmented space" is often adopted to display long paintings, such as Riverside Scene at Qingming Festival. Zigzag composition is often adopted to display landscape of mountains and rivers, showing the gracefulness, smoothness and elegance of Chinese painting.

Western paintings that value realism adopt focus perspective create a striking visual effect through different perspective angles. Such composition values visual center, and generally includes "triangle composition", "ring composition", "diagonal composition", "golden section composition", "slash composition", "V-shape composition", "horizontal composition" and so on. The integration of diverse cultures promotes the fusion of Chinese and Western aesthetic principles in illustration design. The Descendant of Crane ("Fig. 1") Ruan Feifei created as the cover of namesake novel features strong Oriental flavor. Lotus, lotus leaf, crane and auspicious clouds are all common Chinese elements in the work, and the smooth lines endows a sense of fineness. A typical triangular composition is adopted, conveying a stable feeling. In the painting, the crane and the figure are distinct in size. The line between crane, long neck, lotus, and figure is S-shaped, channeling the viewer's attention. 


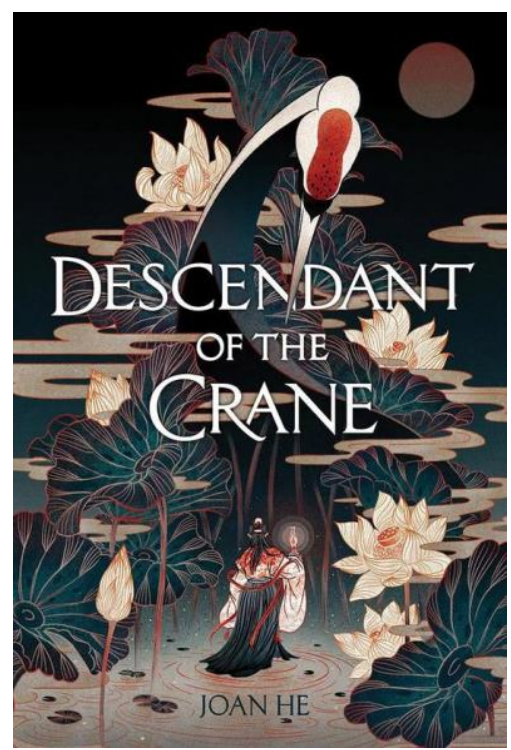

Fig. 1. Cover of Descendant of Crane (from network).

\section{SMART AND SMOOTH LINES}

Both Chinese and Western artists attach great importance to lines, which fully reflects people's perception, generalization and refinement of objective world, and creation which involves people's participation. In fact, there is no line in the real world, and any line can be interpreted as a surface to some extent. However, artists use lines to express the form, texture, structure and emotion based on their perception. Chinese painting attaches great importance to lines. The length, density and weight of lines make the painting full of rhythm. Traditional Chinese realistic painting is shaped by line, which serves as the most basic expression language. The lines and strokes of traditional Chinese realistic painting is shown through ink. Ink is a unique painting tool in oriental art. Its integration with brush pen is ideal, which brings idyllic beauty to people. [3] The combination of brush and ink allows more possibilities to weight, and pause and transition in rhythm of lines in traditional Chinese paintings, which is one of the distinctive features. The analysis of Victo Ngai's Year to Come ("Fig. 2") reveals the huge influence of traditional Chinese realistic painting. She employed lines to create a cheerful rooster, and the chickens on the left and right echoed each other through the smooth S-shaped lines, which made the picture fluid and vivid. The carrier of "elegant" culture also affect the development of blue and white. Chinese painting emphasizes "气韵生动 (lively spirit and charm)" and "形神兼备 (unity of form and spirit)". "气韵" refers to the "air" that thrives all things in the universe, while "形神" emphasizes the similarity in form and spirit, such as the appearance, shape and features of the depicted objects. Calligraphic style of drawing stresses mid tip of the writing brush, side tip of the writing brush, accurate and strong collapse, drawing, and dot. [4] Gu Kaizhi, a famous painter in the Eastern Jin Dynasty, emphasized that painting should "express spirit in form", which means to express the inner spirit and connotation of the object on the basis of accurate form. "Fig. 2" depicts the necks and tails of two roosters with smooth lines. Their feathers have a sense of flow, consistency and hierarchy without stiffness. On the contrary, they appear elegant and unrestrained, which makes people feel comfortable visually. The head is decorated with dots, and the connections between dots, lines and planes enrich the picture, so that the whole picture has a sense of rhythm, which not only reflects the characteristics of the rooster but also shows its charm. Xie He's "Six Techniques of Painting" includes bone method, that is, a painter should seek for inner essence beneath the surface of an object, and should try his best to show inner "essence", "spirit" and "aura" instead of completely copying the appearance when creating.

In terms of line colors, modern illustrations is no striker for traditional black. Some colors are chosen to keep the overall tone consistent, while others are marked with white or gold. This can be explained by more possibilities for designers brought from modern digital products, as well as designers' attention on the consistency, contrast and relativity of color under the influence of Western art. First, the similar colors of lines and the objects depicted can ensure the harmony and unity of the overall tone of the picture. Second, although the designer tries to express beauty through lines, they will not use color that distracts attention. Therefore, the designer will reduce the transparency of the lines to ensure the harmony of the picture during the drawing process. Some works will adopt white and other light colors to mark the subject. Such light colors will make people feel comfortable, and the overall picture will be light. 


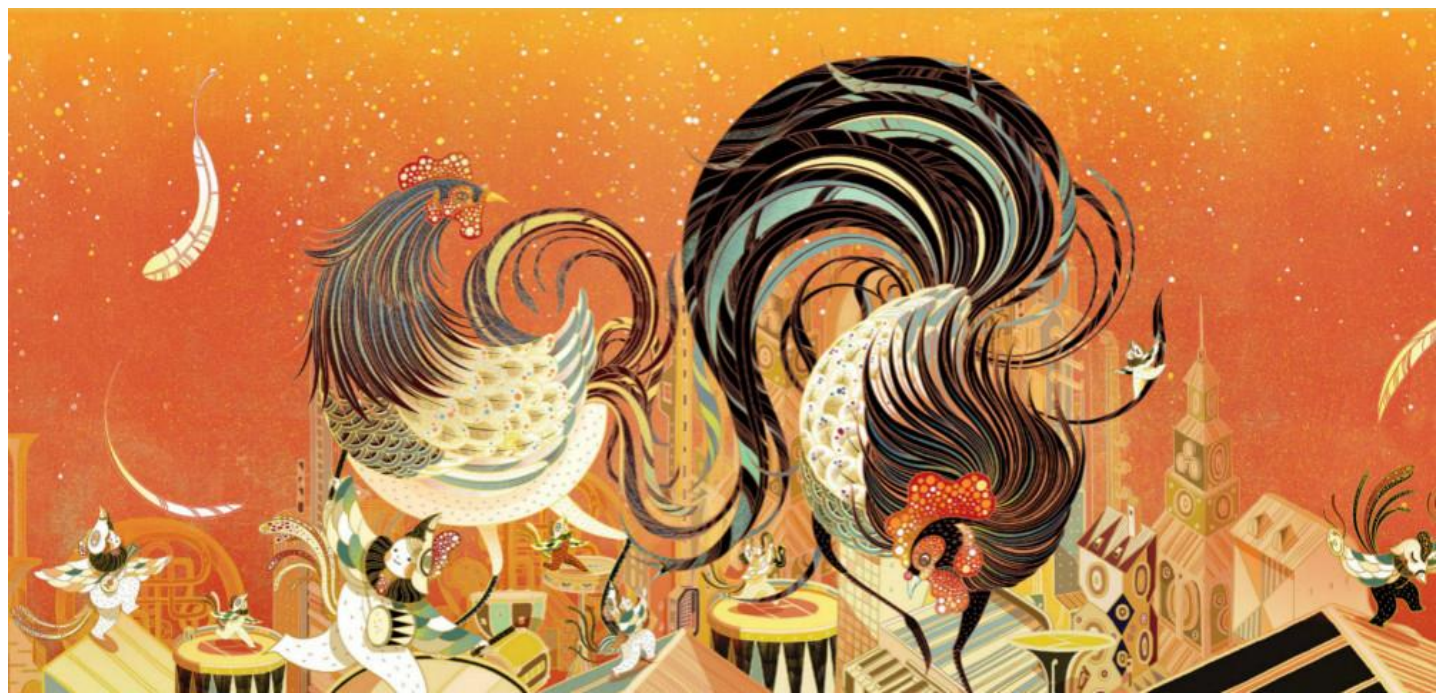

Fig. 2. Year to Come (from network).

\section{MATCH COLOR FOLLOWS THE PRINCIPLE THAT LESS IS MORE}

Color is one of the most expressive visual languages in illustration art creation, which can most directly convey the atmosphere of the picture. [5] Chinese painting follows "color with given categories" in coloring, that is, dividing the objects in the picture into several categories and color them with distinct colors. "Color with given categories" also changed with the development of painting. Thick ink and light ink are used to show green leaves or red flowers. However, ink can also be divided into seven, such as break Chinese ink (a technique of traditional Chinese painting), amass Chinese ink (a technique of traditional Chinese painting), dry ink, leftover ink and splash-ink (a technique of Chinese ink-painting), etc., which can create a kaleidoscope with shades. Thus, color with given categories evolves into ink rendering with given categories.

Modern Chinese style illustration creates color beauty by restricting color, which conforms to the principle of less is more. Color restriction can ensure more impressive visually, more compact, powerful, harmonious, and better color effect. Modern Chinese illustration attaches great importance to comprehensive use of color contrast, consistency, emphasis, and proportion. Color channels attention, highlights the key points expressed, and creates an atmosphere that echoes the theme of the work. The theme can be adopted as a basis for color selection. Master works of ancient and modern inside and outside China, the color matching of exquisite pattern design and the color matching with national style serve as reference. The quantity of color employed should not be too much, in order to ensure the harmony and unify of whole tone. "Fig. 3" is one of Victo Ngai's works. Stable triangular composition is adopted. Smooth S-shaped lines channel people's attention from top to bottom. Complementary colors of red and green are used. In order to keep attention on koi fish in the centre, the most intense complementary contrast is adopted to depict ascending carp and water wave. But a closer look at the color shows that the middle still has the highest color saturation. When the colors in the picture are maintained at a soft saturation, the colors support each other, which helps the designer convey what he or she wants to emphasize. In addition, the overall tone can help the painter render the atmosphere, control the brightness and warmth of the whole picture, and create a mysterious, scary, warm and active atmosphere. In addition, colors echo in the picture can also stabilize the picture, realizing the overall harmony between various parts of the picture.

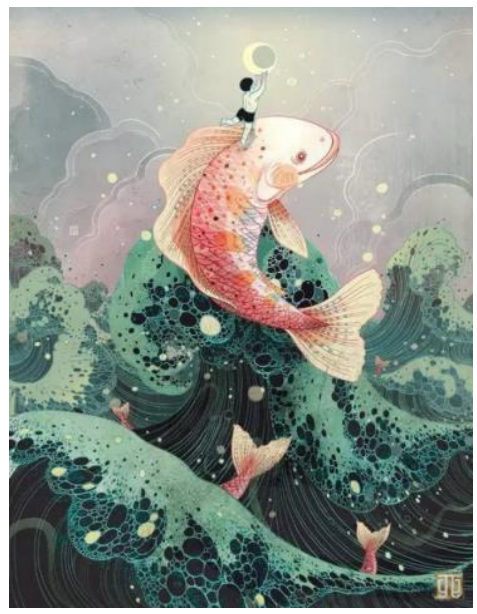

Fig. 3. Koi Fish (from network). 


\section{A MODERN AESTHETIC REDESIGN OF TRADITIONAL ELEMENTS}

In ancient times, Chinese painting was called China blue. After the introduction of western oil painting, it was called Chinese paintings, or traditional Chinese painting to set it apart. With its distinctive characteristics and style, Chinese painting distinguish itself among the world's painting, integrating Chinese people's feelings towards nature, society, philosophy, religion, morality and literature and art. Chinese painting falls into several camps based on themes: figure painting, Chinese landscape painting, bird-andflower paintings, ruler painting. Modern Chinese illustrations are used to exploring representative elements and creative techniques, which is integrated into modern flower arrangement works through design. By virtue of typical elements, designers reveal national characteristics, tell story, and make the picture full and unique.

When creating characters, designers will seek the creative ideas and expression methods of ancient painters from traditional paintings, and combine them with the current era and their own ideas. In this way, the characters created can not only reflect the characteristics of their own nation, but also provide modern people with the enjoyment of beauty. "Fig. 4" is the Chubby Face re-created by the illustrator Jiao Xiangyue based on "White Stone Mural Painting of Musicians and Dance". The designer exaggerated plump beauty fashioned in the Tang Dynasty, combined with the modern animation method to design plump and charmingly naive characters. As the original stone carving is only used for burial, the overall atmosphere of the picture is less joyful and more serious. Different characters in the picture seems to play seriously. The series created by Jiao Xiangyue not only follows the characteristics of the original in expression, but also adds details to each person's expression through the creation of modern cartoon images, so that the viewer can feel that each character in the picture has different inner activities and personality during the performance process. The figures depicted in the paintings are either absorbed in their own performance, struggling to beat the drums, or quietly observing others. The figure also adopts the smooth lines in the original drawing, echoing the rounded figure on the whole. As to character dynamics, the designer first follows the movements of the characters in "White Stone Mural Painting of Musicians and Dance", changes the head and body proportion of the characters, and exaggerates the fullness of each character, making the picture more vivid and interesting. In terms of color, the overall color follows the reddish and yellow tones in Beauties Wearing Flowers, on the basis of which the lightness and purity of the colors are enhanced, making the picture more lively. The original depicts the scene in which music is played for the dead, presenting a sense of rigor. The work created by Jiao Xiangyue can make viewers feel that people sitting on the right side of the picture watching the performance are very happy and do some celebratory activities together.

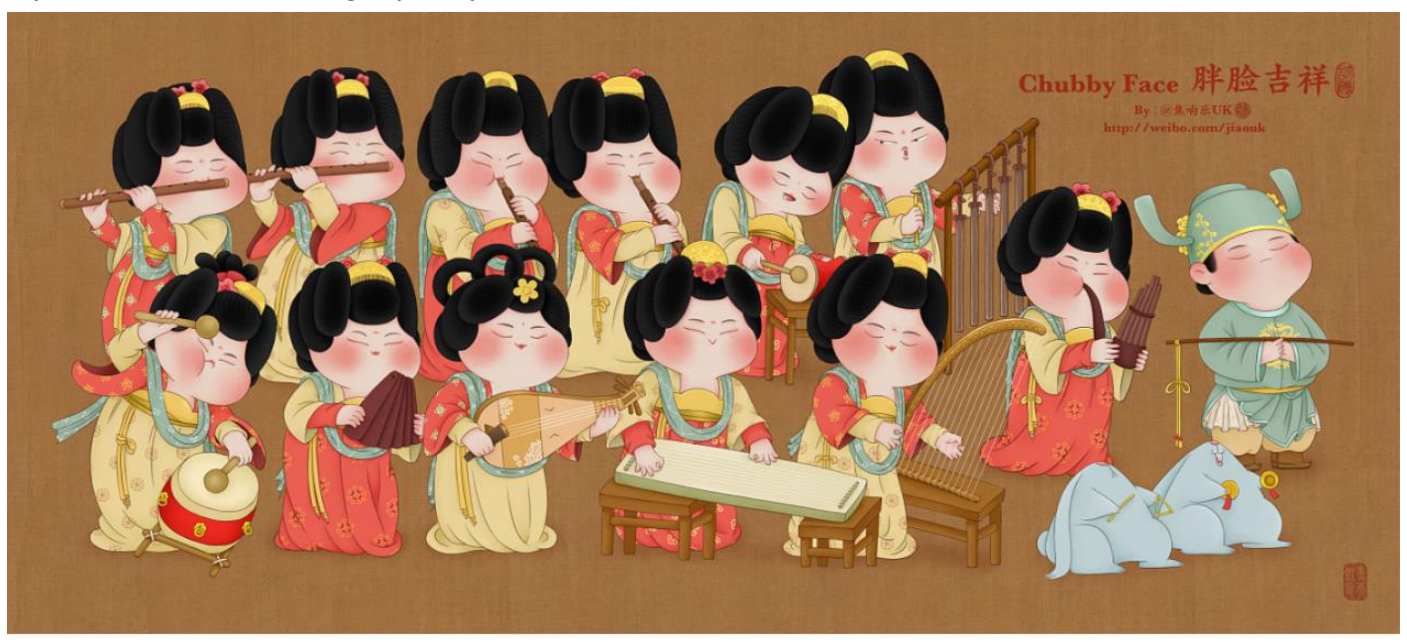

Fig. 4. White Stone Mural Painting of Musicians and Dance (from network).

In addition to figures, common Chinese elements in modern illustrations include landscape, auspicious clouds, aged-pine, cranes, koi, lotus, dragon and phoenix, and typical Chinese ancient buildings. These Chinese elements are interspersed in the picture to create the unique artistic conception and charm of Chinese painting. When creating illustrations, some designers are more loyal to traditional painting methods, while more refine and summarize the modeling, treat the color on the basis of the ancients, keeping its charm with modern simple fashion. Chinese elements in series poster works with Chinese style that Ruan Feifei created for BBC's Doctor Who ("Fig. 5") reveal that the overall atmosphere of the picture is 
elegant and smart, with the vividness and charm emphasized in Chinese paintings. However, careful observation of the elements shows that Ruan Feifei values the sense of order. In "Fig. 5", the light and shade of the water in the waterfall behind the panda varies with length and color. The waves of the water in front are also shaped by concise and smooth lines. Such abstract and concise expression follows the principle of design: elements are highly refined and abstracted. This endows the image antique and modernity in style and simplicity. The image of the rock is also redesigned. The stone in traditional Chinese painting emphasizes the corner angle of line, as well as the contrast between thickness and density, and it is texturized by wrinkle method in the gray area. The rocks in "Fig. 5" are highly generalized and rounded. The thickness of the lines is unified, and the relationship between light and shade is expressed through density and superposition. Wrinkle method has been eliminated, and the smooth transition of color is used to create a sense of volume and the relationship between the primary and secondary expression. Such elaborately designed concise expression makes the picture as a whole, clean and neat but not rigid, fashionable and with distinctive national characteristics.

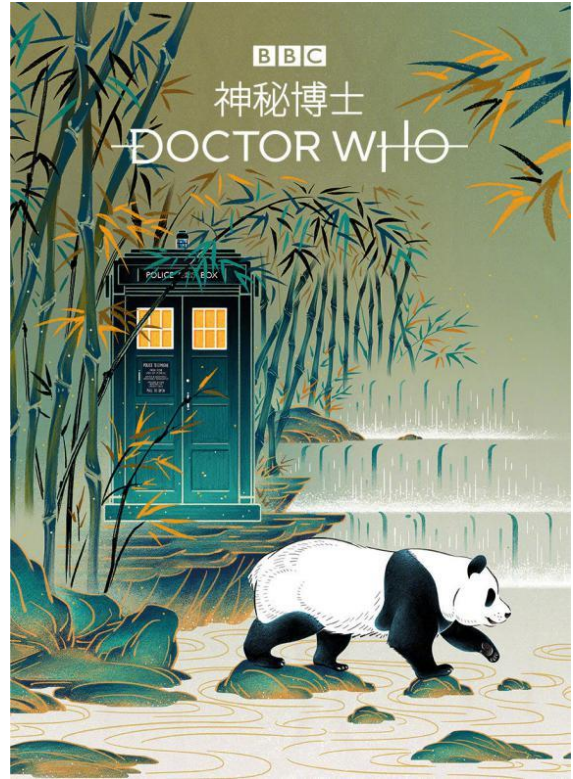

Fig. 5. Doctor Who (from network).

\section{CONCLUSION}

Illustration design is different from art creation. Practical illustration design is the self-expression of the creator, which needs to be appreciated and understood by more laymen, so as to achieve the purpose of commercial or brand communication. In such an era of highly inclusive multi-culture, developed network communication, and rapid information exchange, besides audiences within its country, enterprises or brands attempt to attract groups with various cultural backgrounds, in a bid to promote commercial and cultural communication. Therefore, an illustration work contains both typical Chinese elements and connotation, and Western painting techniques and expressions, which enable people across the world to understand the work and find cultural identity, thus expanding the scope and intensity of communication.

The birth and development of each kind of art has its origin, as well as enduring vitality. [6] Chinese artistic concept benefits from accumulation of thousands of years, the exchange of different nationalities for a long time, and the integration of Chinese philosophical system of Confucianism, Buddhism and Taoism. The collision between Chinese and Western cultures enables people of other nationalities to have a more intuitive understanding of Chinese traditional culture and aesthetic preference, which not only serves as an artistic exchange and innovation, but also breaks down the barriers between nations in cultural, ideological and philosophical thinking and attitudes towards life to a certain extent. This is not only the inheritance and development of traditional culture, but also a milestone in the history of art.

\section{References}

[1] Zhang Jing. Research on the function of artistic language as the medium of aesthetic creation [J]. Theoretical Studies In Literature and Art, 2011(01):73. (in Chinese)

[2] Ding Lingping. On the composition in the creation of traditional Chinese bird-and-flower paintings [J]. Arts In China, 2013,(1):188. (in Chinese)

[3] Sun Lei. The Application of Traditional Chinese Realistic Painting Techniques in Digital Illustration Design [J]. Art And Design, 2013,2(03):109-110. (in Chinese)

[4] Lin Lu. A Brief Talk on Blue and White Decoration and Formal Beauty [J]. Jingdezhen's Ceramics, 2009(2): 31-32. (in Chinese)

[5] Zheng Huizhi, Luo Xiaohuan. The Collision and Fusion of Oriental Charm and Western Art: Analysis of Victo Ngai's Illustration [J]. Design Research, 2018,8(05):28-31. (in Chinese)

[6] Chen Lan. Illustrator [M]. Shanghai: Shanghai Literature and Art Publishing House, 2008. (in Chinese) 